\title{
Two-Phase Concurrent Sensing and Transmission Scheme for Full Duplex Cognitive Radio
}

\author{
Shree Krishna Sharma*, Tadilo Endeshaw Bogale ${ }^{\dagger}$, Long Bao Le ${ }^{\dagger}$, Symeon Chatzinotas*, \\ Xianbin Wang ${ }^{\ddagger}$, Björn Ottersten* \\ ${ }^{*}$ SnT - securityandtrust.lu, University of Luxembourg, Luxembourg \\ Email: \{shree.sharma, symeon.chatzinotas, bjorn.ottersten\}@uni.lu \\ ${ }^{\dagger}$ INRS, Université du Québec, Montréal, QC, Canada, Email: \{tadilo.bogale, long.le \}@emt.inrs.ca \\ $\ddagger$ University of Western Ontario, London, ON, Canada, Email: xianbin.wang@uwo.ca
}

\begin{abstract}
Among several potential applications of Full-Duplex (FD) technology, FD Cognitive Radio (CR) communication is one important area where FD can provide several advantages and possibilities such as concurrent sensing and transmission, improved sensing efficiency and the secondary throughput. However, the main challenge is to mitigate the harmful effects of the residual Self-Interference (SI) which depends on the SI mitigation capability of the employed technique. One way to mitigate this effect is to control the transmit power of the CR node, however, this power control over the entire frame duration results in a power-throughput tradeoff. In this context, we propose a novel Two-Phase Concurrent Sensing and Transmission (2P-CST) framework in which a CR performs concurrent sensing and transmission for a certain fraction of the frame duration by employing a power control mechanism and for the remaining fraction of the frame duration, the CR only transmits with the full power. The proposed framework allows the flexibility to optimize the sensing time and the transmit power in order to maximize the achievable throughput of the CR system. Our results demonstrate that the proposed 2P-CST FD transmission strategy provides better performance in terms of the achievable throughput than the conventional Periodic Sensing and Transmission (PST) and CST techniques.
\end{abstract}

\section{INTRODUCTION}

In contrast to the conventional belief that a radio node can only operate in a Half-Duplex (HD) mode on the same radio channel because of the Self-Interference (SI), it has been recently shown that Full-Duplex (FD) technology is feasible and it can be a promising candidate for the fifth generation (5G) of wireless communications [1], [2]. In general, an FD system can provide several advantages such as potential doubling of the system capacity, reducing end-end/feedback delays, increasing network efficiency and spectrum utilization efficiency [2]. Besides, recent advances in different SI cancellation techniques such as antenna cancellation, analog cancellation and digital cancellation methods have led to the feasibility of using FD technology in different wireless applications. However, due to various practical imperfections and the incapability of the employed SI mitigation schemes, the effect of residual SI on the system performance is a crucial aspect to be considered while incorporating FD technology.

Recently, applications of Full-Duplex (FD) technology in Cognitive Radio (CR) communications, which enables the spectral coexistence of different wireless networks with the help of dynamic spectrum access or spectrum sharing [3], have received significant attention [4]-[6]. In order to enable CR communications, different transmission strategies have been proposed with the objectives of enhancing the sensing efficiency and throughput of the secondary system while protecting primary systems. In this context, a sensing-throughput tradeoff for the Periodic Sensing and Transmission (PST) based approach in an HD CR, in which the total frame duration is divided into two slots (one slot dedicated for sensing the presence of Primary Users (PUs) and the second slot reserved for secondary data transmission) has been studied in several literature [7], [8]. On the other hand, a Full-Duplex (FD) transmission strategy such as Listen And Talk (LAT) [5], which enables simultaneous sensing and transmission at the CR node, can overcome the performance limit due to the HD sensing-throughput tradeoff. However, the main problem is that sensing performance of the FD-CR degrades due to the effect of the residual SI.

One way of mitigating the effect of residual SI on the sensing performance of a CR node is to employ a suitable power control mechanism. In this context, existing contributions have considered Concurrent Sensing and Transmission (CST) method [5] in which the CR node needs to control its transmission power over the entire frame duration. However, this results in a power-throughput tradeoff which arises due to the fact that the employed power control results in the reduction of the SI effect on the sensing efficiency but the secondary throughput is limited. This subsequently results in a power-throughput tradeoff problem for an FD-CR node [5]. The main assumption behind this approach is that the FDCR node transmits with the controlled power over the entire frame duration and it does not consider the optimization of the sensing time.

In the above context, we propose a novel Two-Phase CST (2P-CST) transmission framework in which the FD-CR node performs Spectrum Sensing (SS) for a certain fraction of the frame duration and also transmits simultaneously with the controlled power. For the remaining fraction of the frame duration, the CR only transmits with the full power. In this way, we have the flexibility of optimizing both the parameters, i.e., sensing time and the transmit power in the first slot with the objective of maximizing the secondary throughput. Subsequently, we carry out the performance analysis of the 


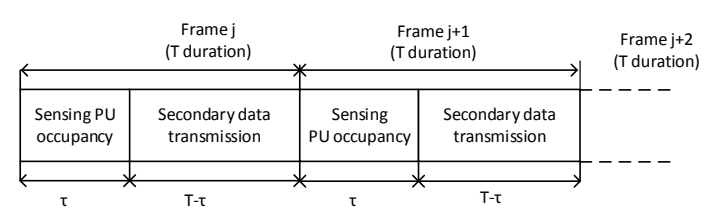

(a)

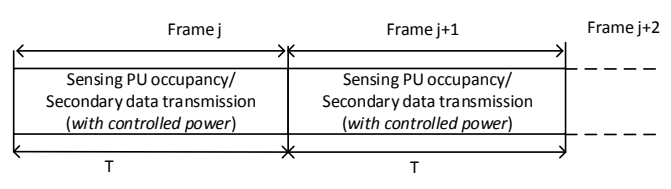

(b)

Fig. 1. Secondary frame structure for (a) PST, (b) CST

proposed method and compare its performance with that of the conventional PST and CST strategies.

The remainder of this paper is organized as follows: Section II presents a system model and also describes the frame structures for the conventional PST, CST and the proposed 2P-CST schemes. Section III analyzes the performance of the proposed scheme in terms of the achievable secondary throughput. Subsequently, Section IV evaluates and compares the performance of proposed method with the conventional schemes via numerical results. Finally, Section V concludes this paper.

\section{Signal Model and TRANSMission Schemes}

The received signal at the FD-CR node under the hypotheses of the PU signal presence $\left(H_{1}\right)$ and the PU signal absence $\left(H_{0}\right)$ can be expressed as [9]

$$
r[n]=\left\{\begin{array}{l}
\sqrt{\eta} s_{\mathrm{i}}[n]+s_{\mathrm{p}}[n]+w[n], \\
\sqrt{\eta} s_{\mathrm{i}}[n]+w[n], \quad n=1, \cdots, N \quad H_{0}
\end{array}\right.
$$

where $s_{\mathrm{i}}[n]$ is the self-transmitted signal, $s_{\mathrm{p}}[n]$ is the PU transmitted signal, $w[n]$ is the additive white Gaussian noise, $\eta$ represents the capability of an FD-CR to mitigate the SI effect, and $N$ is the number of acquired samples within a sensing duration. If $\eta=0$, the CR can cancel the SI completely, otherwise, it can only mitigate its effect. In order to detect the presence (absence) of $s_{\mathrm{p}}[n]$, we employ an Energy Detector (ED) under the assumption that $s_{\mathrm{i}}[n], s_{\mathrm{p}}[n], w[n]$ are independent and identically distributed (i.i.d.) Gaussian random variables. Under such assumption, one can treat $\sqrt{\eta} s_{\mathrm{i}}[n]+w[n]$ as an independent random variable with variance $\sigma^{2}+\eta \mathrm{E}\left\{\left|s_{\mathrm{i}}[n]\right|^{2}\right\}$.

In the following, we describe the frame structures for the conventional schemes and the proposed 2P-CST scheme.

\section{A. Conventional Schemes}

1. Periodic Sensing and Transmission (PST): In this approach (see Frame structure in Fig. 1(a)), the CR operates in a time-slotted mode, i.e., the CR sensing module performs SS for a short duration, let us denote by $\tau$ and transmits data for the remaining $(T-\tau)$ duration, $T$ being the duration of a

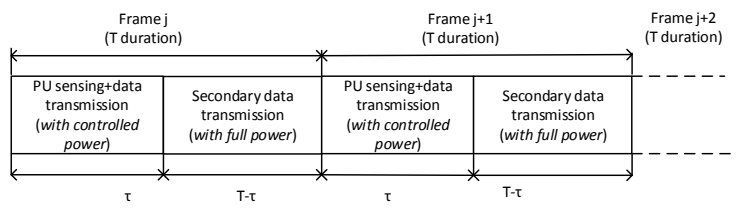

Fig. 2. Secondary frame structure for proposed 2P-CST scheme

frame [7]. The assumption here is that the PU status remains constant over a single frame duration. In practice, either synchronization is required between primary and secondary transmissions or the SU frame must be much shorter than the PU frame for the above assumption to be true. Furthermore, SUs are not able to monitor the PU's status when they are transmitting, hence causing interference to the primary receiver. With this approach, there exists an inherent tradeoff between sensing time and the secondary throughput as noted in various literature [7], [10], [11].

2. Concurrent Sensing and Transmission (CST): In this approach (see Frame structure in Fig. 1(b)), continuous sensing can be achieved and finding an optimal sensing time becomes no longer an issue. However, there exists the problem of residual SI which may degrade the sensing performance. In contrast to the PST approach where the secondary throughput increases with the power monotonously, there exists a powerthroughput tradeoff with this approach, which creates a fundamental limitation in the performance of an FD-CR [5].

B. Proposed Two-Phase Concurrent Sensing and Transmission (2P-CST)

The main drawback of the conventional CST scheme is that the CR needs to control its transmission power in order to mitigate the effect of the SI. If the transmission power is larger than a certain limit, it will affect the sensing performance. For a fixed self-interference suppression quality, sensing performance degrades with the increase in the transmit power due to the increase in the SI. The increased SI causes higher probability of false alarm, thus causing the severe waste of the spectrum opportunities. On the other hand, if the transmit power is small, SI becomes negligible and sensing results become reliable. However, the secondary throughput is limited. Thus, there exists an optimum transmit power which results in the maximum throughput, leading to the power-throughput tradeoff [12].

Regarding the aforementioned power-throughput tradeoff problem, the assumption in most of the related literature is that the CR transmits with the controlled power over the entire frame duration. In this case, power control over the entire frame duration must be performed to mitigate the effect of SI in the sensing performance. To this end, we propose a novel Two-Phase CST (2P-CST) frame structure presented in Fig. 2 in which the transmission strategy can be described as follows: At the beginning of the frame, CR performs SS for a certain fraction of the frame duration and also transmits simultaneously with the controlled power and for the remaining fraction of the frame duration, the CR only transmits with the full power. In this context, our design 
objective is to optimize two parameters: sensing time, and the transmit power in the first slot, which result in the maximum secondary throughput.

\section{PERFORMANCE ANALYSIS}

\section{A. Performance Metrics with Self-Interference}

The commonly used metrics for evaluating the performance of a detector are probability of false alarm $\left(\mathcal{P}_{f}\right)$ and probability of detection $\left(\mathcal{P}_{d}\right)$. Subsequently, using these probabilities, the performance of a CR system can be characterized in terms of different tradeoffs such as sensing-throughput tradeoff and power-throughput tradeoff. As mentioned earlier, there exists a sensing-throughput tradeoff for an HD-CR and a powerthroughput tradeoff for an FD-CR. For the proposed 2P-CST, there exist both the aforementioned tradeoffs and we can characterize its performance in terms of the sensing-powerthroughput tradeoff.

Regarding the binary hypothesis testing problem in (1), the test statistic $(D)$ for an ED is given by

$$
D=\frac{1}{N} \sum_{n=1}^{N}|r(n)|^{2}
$$

In (2), $D$ is a random variable and its Probability Density Function (PDF) under the $H_{0}$ hypothesis follows a Chisquared distribution with $2 N$ degrees of freedom for the complex valued case. For very large values of $N$, the PDF of $D$ can be approximated by a Gaussian distribution with mean $\mu=\sigma_{w}^{2}$ and the variance $\sigma_{0}^{2}=\frac{1}{N}\left[E[w(n)]^{4}-\sigma_{w}^{2}\right]$ [7], where $E[$.$] denotes an expectation operator. The expressions for \mathcal{P}_{f}$ and $\mathcal{P}_{d}$ can be computed by; $\mathcal{P}_{f}=\operatorname{Pr}\left(D>\lambda \mid H_{0}\right)$, and $\mathcal{P}_{d}=$ $\operatorname{Pr}\left(D>\lambda \mid H_{1}\right)$, where $\lambda$ is the decision threshold. We consider the case of the circularly symmetric complex Gaussian noise case in which $E[w(n)]^{4}=2 \sigma_{w}^{4}$, thus $\sigma_{0}^{2}=\frac{1}{N} \sigma_{w}^{4}$.

For the conventional CST scheme, the probability of false alarm $\mathcal{P}_{f}$ is related to the target probability of detection $\overline{\mathcal{P}}_{d}$ as follows [7]

$$
\mathcal{P}_{f}=Q\left(\sqrt{\left(2 \gamma_{p}+1\right)} Q^{-1}\left(\overline{\mathcal{P}}_{d}\right)+\sqrt{\tau f_{s}} \gamma_{p}\right),
$$

where $Q($.$) is the complementary distribution function of$ the standard Gaussian random variable, $\gamma_{p}$ is the PU SNR measured at the secondary transmitter, $\tau$ is the sensing time and $f_{s}$ is the sampling frequency.

The main problems in the conventional PST scheme are that transmission slot needs to be divided into small discontinuous time slots even if the spectrum opportunity is available for a long period, and SUs cannot monitor the changes of PUs' states, during data transmission phase, which leads to the collision when the PUs become active and the spectrum opportunity is wasted when PUs become inactive [5].

As discussed earlier, the main problem with the CST strategy in an FD-CR is that the node suffers from the SI due to its own transmitted signal, hence causing the sensing errors. The expression for $P_{f}$ for an FD transceiver depends on the following cases, namely, perfect and imperfect SI cancellation.
1. Without Residual Self-Interference (Perfect SI Cancellation): For a target $\overline{\mathcal{P}}_{d}, \mathcal{P}_{f}$ in (3) for the considered ED technique can be written as

$$
\mathcal{P}_{f}(T)=Q\left(\sqrt{\left(2 \gamma_{p}+1\right)} Q^{-1}\left(\overline{\mathcal{P}}_{d}\right)+\sqrt{T f_{s}} \gamma_{p}\right)
$$

2. With Residual Self-Interference (Imperfect SI Cancellation): Although several antenna-based, RF and digital interference mitigation techniques have been investigated in the literature to mitigate the SI [13], [14], there still remains its residual effect. The sensing-throughput tradeoff performance of the FD transceiver is affected by this residual SI which depends on the SI mitigation capability. This is due to the effect of residual SI on $\mathcal{P}_{d}$ and $\mathcal{P}_{f}$. Considering the residual SI mitigation capability $\eta$ defined in Section II with $\eta \in\{0,1\}$, the expressions for $P_{d}$ and $P_{f}$ can be written as [15]

$\mathcal{P}_{d}(\lambda, \tau)=Q\left(\left(\frac{\lambda}{\sigma_{\mathrm{w}}^{2}}-\eta^{2} \sqrt{\frac{T f_{s}}{2 \eta^{2} \gamma_{\mathrm{in}}+2 \eta^{2} \gamma_{\mathrm{in}} \gamma_{\mathrm{p}}+2 \gamma_{\mathrm{p}}+1}}\right)\right)$

$$
\mathcal{P}_{f}(\lambda, \tau)=Q\left(\left(\frac{\lambda}{\sigma_{w}^{2}}-\eta^{2} \gamma_{\mathrm{in}}-1\right) \sqrt{\frac{T f_{s}}{2 \eta^{2} \gamma_{\mathrm{in}}+1}}\right),
$$

where $\gamma_{\text {in }}$ denotes the ratio of the strength of the SI to the noise power, measured at the receiver of the same node. Combining (5) and (6), the expression for $\mathcal{P}_{f}$ for a target $\overline{\mathcal{P}}_{d}$ can be written as

$$
\begin{gathered}
\mathcal{P}_{f}=Q\left(\left(Q^{-1}\left(\overline{\mathcal{P}}_{d}\right) \sqrt{2 \eta^{2} \gamma_{\mathrm{in}}+2 \eta^{2} \gamma_{\mathrm{in}} \gamma_{\mathrm{p}}+2 \gamma_{\mathrm{p}}+1}\right.\right. \\
\left.\left.\left.+\gamma_{\mathrm{p}} \sqrt{T f_{s}}\right) \frac{1}{\sqrt{2 \eta^{2} \gamma_{\mathrm{in}}+1}}\right)\right) .
\end{gathered}
$$

\section{B. Tradeoff Analysis}

We denote the full secondary transmit power by $P_{\text {full }}$, the controlled secondary power by $P_{\text {cont }}$, and the PU transmit power by $P_{\mathrm{p}}$. The expressions for the throughput of the secondary network in the absence $\left(C_{0}\right)$ and the presence $\left(C_{1}\right)$ of the active PU can be defined as

$$
\begin{aligned}
C_{0} & =\log _{2}\left(1+\gamma_{s}\right) \\
C_{1} & =\log _{2}\left(1+\frac{\gamma_{s}}{1+\gamma_{p}}\right) .
\end{aligned}
$$

Let $\mathcal{P}\left(H_{0}\right)$ denote the probability of the PU being inactive, and $\mathcal{P}\left(H_{1}\right)$ as the probability of the PU being active. When there is perfect detection under the $H_{0}$ hypothesis, i.e., $\mathcal{P}_{f}=0$, then the throughput of the secondary link is given by $\frac{T-\tau}{T} C_{0}$. Since there always exists some non-zero probability of false alarm $\mathcal{P}_{f}$ in practice, the probability of having perfect detection under the $H_{0}$ hypothesis is given by $\left(1-\mathcal{P}_{f}(\lambda, \tau)\right) \mathcal{P}\left(H_{0}\right)$ [7]. Similarly, under the $H_{1}$ hypothesis, the throughput of the secondary link under the ideal case is given by $\frac{T-\tau}{T} C_{1}$ and the probability of having such a situation can be written as: $\left(1-\mathcal{P}_{d}(\lambda, \tau)\right) \mathcal{P}\left(H_{1}\right)$.

For the conventional PST approach, the average throughput for the secondary network is given by

$$
R_{\mathrm{PST}}(\lambda, \tau)=R_{0}(\lambda, \tau)+R_{1}(\lambda, \tau)
$$


where the values of $R_{0}(\lambda, \tau)$ and $R_{1}(\lambda, \tau)$ can be calculated using the following expressions

$$
\begin{aligned}
& R_{0}(\lambda, \tau)=\frac{T-\tau}{T}\left(1-\mathcal{P}_{f}(\lambda, \tau)\right) \mathcal{P}\left(H_{0}\right) C_{0}, \\
& R_{1}(\lambda, \tau)=\frac{T-\tau}{T}\left(1-\mathcal{P}_{d}(\lambda, \tau)\right) \mathcal{P}\left(H_{1}\right) C_{1},
\end{aligned}
$$

where the values of $C_{0}$ and $C_{1}$ are obtained from (8), with $\gamma_{\mathrm{s}}=\frac{P_{\text {full }}}{N_{0}}$, and $\gamma_{\mathrm{p}}=\frac{P_{\mathrm{p}}}{N_{0}}$, with $N_{0}$ being the noise power measured at the $\mathrm{CR}$ node.

For the CST approach, sensing duration is $T$ instead of $\tau$ in the PST approach. Therefore, the total throughput of the CST approach can be written as [16]

$$
R_{\mathrm{CST}}(\lambda, T)=R_{0}(\lambda, T)+R_{1}(\lambda, T),
$$

where the values of $R_{0}(\lambda, T)$ and $R_{1}(\lambda, T)$ can be calculated using the following expressions

$$
\begin{aligned}
& R_{0}(\lambda, T)=\left(1-\mathcal{P}_{f}(\lambda, T)\right) \mathcal{P}\left(H_{0}\right) C_{0}, \\
& R_{1}(\lambda, T)=\left(1-\mathcal{P}_{d}(\lambda, T)\right) \mathcal{P}\left(H_{1}\right) C_{1},
\end{aligned}
$$

where the values of $C_{0}$ and $C_{1}$ are obtained from (8), with $\gamma_{s}=\frac{P_{\text {cont }}}{N_{0}}$, and $\gamma_{\mathrm{p}}=\frac{P_{\mathrm{p}}}{N_{0}}$.

In the proposed 2P-CST scheme, the total throughput will be contributed both from the controlled power and full power transmissions. In this context, the additional throughput, let us denote by $R_{2}$, is given by

$$
R_{2}(\lambda, \tau)=\frac{\tau}{T}\left(1-\mathcal{P}_{f}(\lambda, \tau)\right) \mathcal{P}\left(H_{0}\right) C_{0}+\frac{\tau}{T}\left(1-\mathcal{P}_{d}(\lambda, \tau)\right) \mathcal{P}\left(H_{1}\right) C_{1}
$$

where the values of $C_{0}$ and $C_{1}$ are obtained from (8), with $\gamma_{s}=\frac{P_{\text {cont }}}{N_{0}}$, and $\gamma_{\mathrm{p}}=\frac{P_{\mathrm{p}}}{N_{0}}$.

For the proposed $2 \mathrm{P}$-CST scheme, we formulate the throughput optimization problem in two ways as follows:

i. Approach 1: In this scheme, the controlled power $P_{\text {cont }}$ is calculated based on the SI mitigation capability $\eta$. Based on this model, the controlled power is calculated as

$$
P_{\text {cont }}=P_{\text {full }}(1-\eta) \text {. }
$$

From (14), it is implied that since $\eta$ varies from 0 and $1, P_{\text {cont }}$ varies from $P_{\text {full }}$ to 0 .

The optimization problem for this approach can be written as

$$
\begin{gathered}
\max _{\tau} R_{2 \mathrm{PCST}}(\lambda, \tau)=R_{0}(\lambda, \tau)+R_{1}(\lambda, \tau)+R_{2}(\lambda, \tau), \\
\text { subject to } \mathcal{P}_{d}(\lambda, \tau) \geq \overline{\mathcal{P}}_{d},
\end{gathered}
$$

where $R_{0}(\lambda, \tau)$ and $R_{1}(\lambda, \tau)$ can be obtained using (10) and $R_{2}(\lambda, \tau)$ using (15). This approach allows us to make the fair comparison of the proposed approach with the CST approach.

ii. Approach 2: In this method, the controlled power is not based on the value of $\eta$ and we optimize both parameters $P_{\text {cont }}$ and $\tau$. The secondary throughput optimization problem for this case can be formulated as

$$
\begin{array}{r}
\max _{\tau, P_{\text {cont }}} R_{2 \operatorname{PCST}}(\lambda, \tau)=R_{0}(\lambda, \tau)+R_{1}(\lambda, \tau)+R_{2}(\lambda, \tau), \\
\text { subject to } \mathcal{P}_{d}(\lambda, \tau) \geq \overline{\mathcal{P}}_{d},
\end{array}
$$

where $R_{0}(\lambda, \tau)$ and $R_{1}(\lambda, \tau)$ are obtained from (10) and $R_{2}(\lambda, \tau)$ from (13).

To solve the optimization problem (16), we take the following iterative approach:

1) For a fixed value of $\eta$, calculate the controlled power based on the first approach (Approach 1).

2) Based on the controlled power in step (1), calculate the optimum value of $\tau$ which provides the maximum throughput.

3) Increment the controlled power in step (1) by $\delta$ and calculate the value of total throughput $R$.

4) Repeat step (3) till the calculated throughput becomes less than or equal to the throughput in the previous iteration and note the corresponding controlled power as the optimum controlled power.

5) Using the optimum controlled power calculated in step (4), calculate $R_{2 \mathrm{PCST}}$.

\section{NumERICAL RESUlTS}

In this section, we present numerical results for evaluating the performance of the conventional PST, CST and the proposed 2P-CST schemes. For this performance evaluation, we consider carrier bandwidth and sampling frequency to be 6 MHz. Let us consider $\mathcal{P}\left(H_{1}\right)=0.2$ and the $\overline{\mathcal{P}}_{d}$ be 0.95 . Unless otherwise stated, we consider the primary received SNR of $-20 \mathrm{~dB}$, frame duration of $0.2 \mathrm{~s}$ and secondary transmit SNR of $10 \mathrm{~dB}$ when FD-CR node transmits with the full power. Besides, we consider a fixed channel attenuation of $10 \mathrm{~dB}$ for the channel between ST and the SR.

Figure 3(a) shows the secondary throughput versus $\tau$ for the PST approach. It can be depicted that there exists a tradeoff between the secondary throughput and sensing time for the PST approach as noted in [7]. It can be further noted that the secondary throughput increases for the higher received power at the secondary receiver. Figure 3(b) depicts the secondary throughput versus transmit SNR for the CST scheme for different values of $\eta$. It can be observed that for $\eta=0$, i.e., perfect SI cancellation, the secondary throughput increases with the increase in the value of transmit SNR. However, in practice, it is impossible to completely suppress the SI and we need to take the residual SI into account. From Fig. 3(b), it can be noted that for $\eta \neq 0$, the secondary throughput first increases, reaches the maximum point and then decreases. As also illustrated in [5], this result clearly shows the tradeoff between transmit power and the secondary throughput in the presence of residual SI. With the increase in the value of $\eta$, the secondary throughput decreases due to the effect of SI and the optimal tradeoff point appears at the lower values of SNR.

In order to analyze the performance of the proposed two 2P-CST approaches, we plot the secondary throughput versus $\tau$ in Fig. 4. It can be deduced that there exists a tradeoff between sensing time and the secondary throughput as in the traditional PST approach. More importantly, the optimum value of throughput due to both approaches is higher than the throughput that can be obtained with the CST method. Furthermore, the optimum throughput for the second approach 


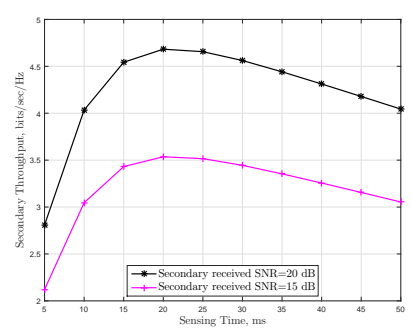

(a)

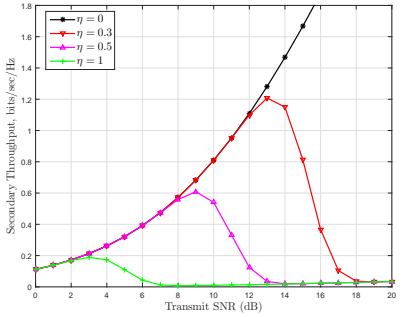

(b)
Fig. 3. (a) Sensing-throughput tradeoff for the PST approach, (b) Powerthroughput tradeoff for the CST method

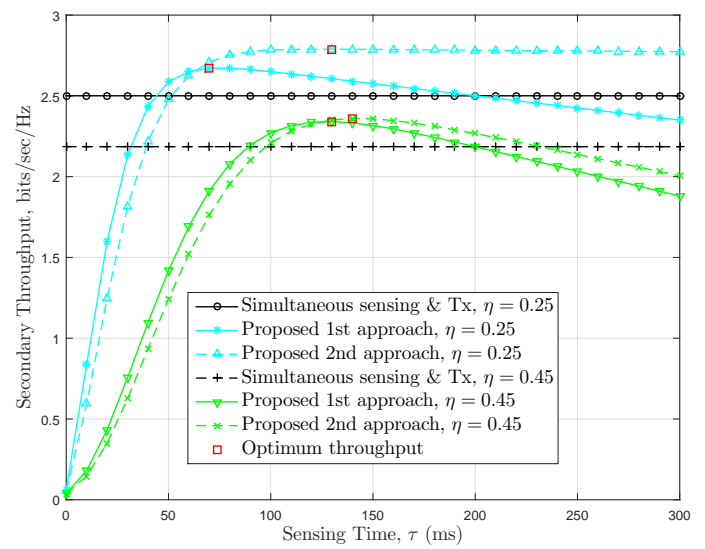

Fig. 4. Secondary throughput versus sensing time for the proposed methods

is higher than the optimum value of the throughput that can be achieved with the first approach for the considered values of $\eta$.

In order to demonstrate the effect of $\eta$ on the optimum throughput provided by the proposed two approaches and by the CST approach, we plot secondary throughput versus $\eta$ in Fig. 5. From the figure, it can be noted that both approaches provide higher throughput than the CST approach. In particular, the proposed second approach provides higher optimum throughput than the first approach up to the value of $\eta=0.5$ and beyond this value, the optimum throughput values of both approaches become the same. On the other hand, the first approach is simple whereas the second approach requires an iterative method to compute the controlled power. Thus, depending on the SI mitigation capability of the FD transceiver and the tolerable implementation complexity, we can make a suitable choice between the proposed techniques.

\section{CONCLUSIONS}

This paper has proposed a novel 2P-CST FD transmission framework by considering a power control mechanism as an important enabler for the FD-CR. The performance of the proposed scheme has been carried out in terms of the achievable secondary throughput by taking the effect of residual SI into account. It has been concluded that the proposed 2P-CST FD transmission strategy provides better performance in terms of

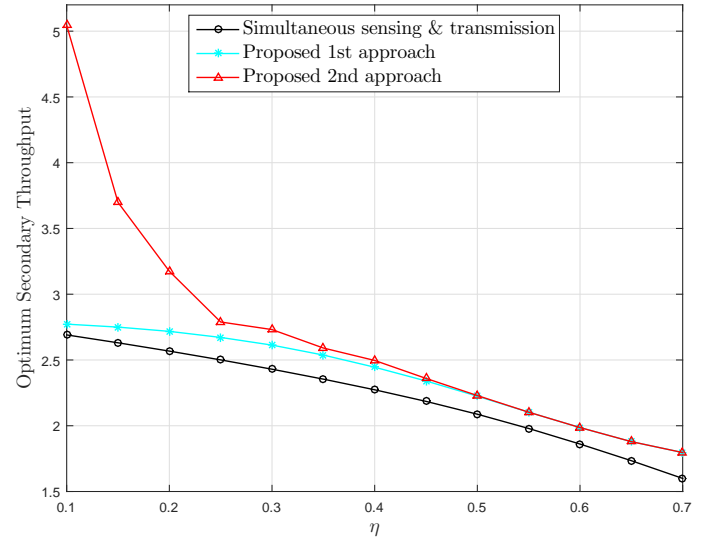

Fig. 5. Secondary throughput versus $\eta$ for the proposed methods

the achievable throughput than the conventional PST and CST techniques.

\section{REFERENCES}

[1] M. Jain et al., "Practical, real-time, full duplex wireless," in Proc. 17th annual int. conf. on Mobile computing and networking, 2011.

[2] S. Hong et al., "Applications of self-interference cancellation in 5G and beyond," IEEE Commun. Magazine, vol. 52, no. 2, pp. 114-121, Feb. 2014.

[3] S. K. Sharma et al. "Cognitive radio techniques under practical imperfections: A survey," IEEE Commun. Surveys Tutorials, vol. 17, no. 4, pp. 1858-1884, Fourthquarter 2015.

[4] J. Yang et al. "Full-duplex spectrum sensing scheme based on phase difference," in Proc. IEEE 80th VTC Fall, Sept. 2014, pp. 1-5.

[5] Y. Liao, L. Song, Z. Han, and Y. Li, "Full duplex cognitive radio: a new design paradigm for enhancing spectrum usage," IEEE Commun. Magazine, vol. 53, no. 5, pp. 138-145, May 2015.

[6] T. Wang, Y. Liao, B. Zhang, and L. Song, "Joint spectrum access and power allocation in full-duplex cognitive cellular networks," in Proc. IEEE Int. Conf. Commun. (ICC), June 2015, pp. 3329-3334.

[7] Y.-C. Liang, Y. Zeng, E. Peh, and A. T. Hoang, "Sensing-throughput tradeoff for cognitive radio networks," IEEE Trans. Wireless Commun., vol. 7, no. 4, pp. 1326-1337, April 2008.

[8] S. Sharma, S. Chatzinotas, and B. Ottersten, "A hybrid cognitive transceiver architecture: Sensing-throughput tradeoff," in Proc. 9th Int. Conf. CROWNCOM, June 2014, pp. 143-149.

[9] X. Yan et al., "Improved energy detector for full duplex sensing," in Proc. IEEE 80th VTC Fall, Sept. 2014, pp. 1-5.

[10] S. Zarrin and T. J. Lim, "Throughput-sensing tradeoff of cognitive radio networks based on quickest sensing," in IEEE Int. Conf. on Commun. (ICC), 2011, pp. 1-5.

[11] M. Cardenas-Juarez and M. Ghogho, "Spectrum sensing and throughput trade-off in cognitive radio under outage constraints over Nakagami fading," IEEE Commun. Letters, vol. 15, no. 10, pp. 1110-1113, Oct. 2011.

[12] Y. Liao, T. Wang, L. Song, and Z. Han, "Listen-and-talk: Full-duplex cognitive radio networks," in Proc. IEEE GLOBECOM, Dec. 2014, pp. $3068-3073$.

[13] E. Ahmed, A. Eltawil, and A. Sabharwal, "Rate gain region and design tradeoffs for full-duplex wireless communications," IEEE Trans. Wireless Commun., vol. 12, no. 7, pp. 3556-3565, July 2013.

[14] M. Duarte, C. Dick, and A. Sabharwal, "Experiment-driven characterization of full-duplex wireless systems," IEEE Trans. Wireless Commun., vol. 11, no. 12, pp. 4296-4307, 2012.

[15] W. Afifi and M. Krunz, "Exploiting self-interference suppression for improved spectrum awareness/efficiency in cognitive radio systems," in Proc. IEEE INFOCOM, Apr. 2013, pp. 1258-1266.

[16] S. Stotas and A. Nallanathan, "On the throughput and spectrum sensing enhancement of opportunistic spectrum access cognitive radio networks," IEEE Trans. Wireless Commun., vol. 11, no. 1, pp. 97-107, Jan. 2012. 